\title{
Rapid acquisition and modulation of colistin-resistance by an extensively drug-resistant Acinetobacter baumannii: case report and review of current literature
}

\author{
Jari Intra, ${ }^{1}$ Roberta M. Sala, ${ }^{1}$ Eduardo Beck, ${ }^{2}$ Paolo Brambilla' \\ ${ }^{1}$ Department of Laboratory Medicine, Milano Bicocca University; ${ }^{2}$ Intensive Care Unit, Desio \\ Hospital, Italy
}

\section{Summary}

Acinetobacter baumannii has emerged as a major cause of healthcare-associated infections. It commonly expresses clinical resistance to multiple antimicrobial agents, and hence, it is considered the paradigm of an extensively drug-resistant (XDR) bacterium. XDR A. baumannii is a rapidly emerging pathogen, especially in the intensive care unit (ICU), causing nosocomial infections including sepsis, ventilatorassociated pneumonia, meningitis, peritonitis, urinary tract infection, and central venous catheter-related infection. In the present report, we described an in vivo evolution of $A$. baumannii strain from a colistinsusceptibility to a colistin-resistance state. A 65 -year-old male, who suffered a duodenal ulcer, two days after hospitalization and during the stay in ICU, contracted a pneumonia and peritoneal infection by a

Correspondence: Jari Intra, Department of Laboratory Medicine, University Milano Bicocca, Desio Hospital, via Mazzini 1, 20833, Desio (MB), Italy. Tel.: +39.0362.3831 - Fax: +39.0362 .3831 .

E-mail: jari.intra@unimi.it

Key words: Gram-negative bacteria; Pandrug-resistance; Nosocomial infection; Colistin; ICU-acquired infection.

Acknowledgments: we gratefully acknowledge Colombo Laura, Savarino Cinzia, Santambrogio Marco, and Caimi Silvio of Desio Hospital for technical support.

Contributions: JI and MRS participated in conception and design of this report and were involved in drafting and revising the manuscript. EB and PB participated in final revision of the manuscript.

Conflict of interest: the authors declare no potential conflict of interest.

Received for publication: 7 March 2016.

Revision received: 2 May 2016.

Accepted for publication: 2 May 2016.

(C) Copyright J. Intra et al., 2016

Licensee PAGEPress, Italy

Microbiologia Medica 2016; 31:5869

doi:10.4081/mm.2016.5869

This article is distributed under the terms of the Creative Commons Attribution Noncommercial License (by-nc 4.0) which permits any noncommercial use, distribution, and reproduction in any medium, provided the original author(s) and source are credited. carbapenem-resistant $A$. baumannii strain. After a combination treatment with colistin, vancomycin plus imipenem, and within seven days, the pathogen rapidly evolved in seven days to a pandrug-resistant phenotype. As the antimicrobial treatment was stopped, the $A$. baumannii isolate changed another time its profile to colistin, becoming newly susceptible, showing a very high level of adaptability to external conditions. We also have reviewed here the current literature on this worryingly public health threat.

\section{Introduction}

Acinetobacter baumannii strains commonly cause a wide spectrum of infection including bacteremia, nosocomial-acquired pneumonia or ventilator-associated pneumonia (VAP), catheter-related infections, meningitis, peritonitis, urinary tract infections, skin and wound infections $(8,9)$. Several studies have confirmed that $A$. baumannii is the most important species involved in the emergence of outbreaks, particularly in intensive care unit (ICUs) and affecting mainly immunocompromised or severely ill patients $(8,9,10,12)$. Various risk factors correlated to colonization and acquisition of $A$. baumannii have been documented, including severity of illness, previous infections or sepsis syndrome, state of colonization with Acinetobacter, recent surgery, previous treatment with broad-spectrum antibiotics, to have been subjected to invasive procedures, presence of central venous or urinary catheter, prolonged mechanical ventilation, and length of ICU and hospital stay $(3,8,9,10,12)$. The associated mortality rate is high, ranging from about 30 to $52 \%$ (3). A. baumannii isolates frequently show increased resistance to multiple antibiotics; this maybe due in part to the relatively impermeable outer membrane, to environmental exposure to a large reservoir of resistance genes, and to the coexistence of multiple mechanisms of resistance $(3,8,9,10,12)$. Multidrug-resistant (MDR) A. baumannii has been reported worldwide often associated with patient-topatient transmission $(3,8,9,10,12)$. The term MDR A. baumannii is normally defined as resistance to one or more agents in at least three classes of antimicrobial categories that would otherwise provide as treatment for Acinetobacter infections (7). Extensively drug resistant (XDR) A. baumannii isolates also show a further resistance to carbepenems, while pandrug-resistant (PDR) A. baumannii isolates are resistant to every US Food and Drug Administration approved antimicrobial agents, including the more toxic agents, such as polymyxins (colstin) and tigecicline (7). Over recent years, nosocomial infection, due to resistant $A$. baumannii strains, has become a common problem in many hospitals in the USA, Europe, and a particularly frequent problem in Asia (3). Here, we report a fatal case involving a patient hospitalised in the Desio Hospital ICU and suffering a duodenal ulcer. Within 
two days, the patient developed an infection caused by a XDR A. baumannii clinical strain that evolved very quickly to a PDR phenotype. The aim of this report is to provide an example of how an A. baumannii strain can modify in vivo its antimicrobial profile against colistin. This resulted in an extension of hospital stay, the appearance of super-infections, multisystem organ failure, and, finally, caused the death of the patient. The study highlights the need to adopt strict infection control measures also when carbapenem-resistant $A$. baumannii isolation appears to be a sporadic event and furthermore, here is presented the current literature about this growing and worrisome problem.

\section{Case Report}

A 65-year-old male, with no remarkable medical history, was admitted to the emergency department of our hospital on August 28, 2015, in an unconscious state. At the moment of his arrival, his vital signs were as follows: blood pressure, 190/95 mmHg; heart rate, 112 beats per minute; respiratory rate, 18 breaths per minute; body temperature, $33.4^{\circ} \mathrm{C}$. He required mechanical ventilation and urgent blood transfusion. In fact, laboratory tests showed a remarkable anaemia with haemoglobin value of $3.8 \mathrm{~g} / \mathrm{dL}$ and haematocrit of $11.9 \%$; white blood cell count of $26,500 / \mathrm{mm}^{3}$; blood glucose and serum creatinine, 310 and $1.03 \mathrm{mg} / \mathrm{dL}$, respectively. A duodenal ulcer was diagnosed. At the time of hospitalization, microbiological cultures were negatives, except the throat swab positive to Candida albicans and a quantitative endotracheal aspirate to Neisseria meningitidis. On August 29, due to the persistence of severe clinical situation and the presence of bleeding after repeated unsuccessful esophagogastroduodenoscopies, the patient underwent a laparotomy, where it was performed a gastrojejunal anastomosis, cholecystectomy with Kehr's t-tube biliary drainage, and a jejunostomy. Extended surgical prophylaxis with piperacillin-tazobactam was started. After three days of ICU stay, the patient resulted colonised by a carbapenemresistant $A$. baumannii strain, identified from both the throat swab and the endotracheal aspirate specimens. The species identification was obtained by Vitek ${ }^{\circledR}$ Matrix-Assisted Laser Desorption Ionization-Time Of Flight Mass Spectrometry system (bioMérieux, Marci-L'Etoile, France). Vitek ${ }^{\circledR} 2$ GN-AST (Antimicrobial Susceptibility Testing) N202 cards were used to gain rapid, accurate susceptibility results and resistance detection. The antimicrobial susceptibility categorisation was done accordingly to EUCAST 2015 clinical breakpoints. Laboratory tests showed an increasing of white blood cell count to $19,900 / \mathrm{mm}^{3}$. Because of high concern about the possible spread of $A$. baumannii in this severely ill patient, treatment with colistin was started on day four. On day six, XDR A. baumannii was also detected from the peritoneal fluid, and the peri-hepatic fluid. Staphylococcus epidermidis and Candida albicans were instead recovered from the pus that appeared near the Kehr. Antibiotic spectrum was widened adding vancomycin, imipenem and fluconazole in addition to colistin. On day 13, peritoneal fluid culture was negative, while an $A$. baumannii strain was another time isolated from the endotracheal aspirate, with changes in its susceptibility profile, showing colistin-resistance. On days 17,19 and 21, respectively, the three surveillance endotracheal aspirate always confirmed the presence of a PDR A. baumannii isolate. Moreover, a blood culture was performed, and it resulted negative. The evolution and improvement of clinical situation of the patient were favourable, and antimicrobial treatment was stopped. However, on day 23, surveillance endotracheal aspirate culture evidenced the presence not only of PDR A. baumannii, but also of another opportunistic and pathogenic gram-negative bacterium often responsible for nosocomial infections, Enterobacter aerogenes. This isolated showed an antimicrobial profile of susceptibility to amikacin, ciprofloxacin, ertapenem, cefepime, gentamicin, imipenem, meropenem, cefotaxime, ceftazidime, and piperacillin/tazobactam, and resist- ance to colistin and amoxicillin/clavulanic acid. On day 24, blood culture and central venous catheter (CVC) culture resulted positive to $E$. aerogenes. On day 25 , surveillance endotracheal aspirate culture evidenced again the presence of both the PDR $A$. baumannii and the $E$. aerogenes strains. Laboratory tests showed an increasing of white blood cell count to $26,200 / \mathrm{mm}^{3}$, and very high values of C-reactive protein and procalcitonin, over $150 \mathrm{mg} / \mathrm{L}$ and over $60 \mathrm{ng} / \mathrm{mL}$, respectively. Antimicrobial treatment with piperacillin-tazobactam was again started. On day 28, surveillance BAL culture remained positive for A. baumannii, E. aerogenes, and, in addition, Staphylococcus aureus. Surprisingly, A. baumannii strain changed its susceptibility to colistin, becoming susceptible. On day 32 , surveillance endotracheal aspirate culture gave positivity for a colistin-susceptible $A$. baumannii strain. Mechanical ventilation was stopped, and spontaneous breathing has been restored. After two days, the patient was discharged from ICU. On day 49, blood culture resulted again positive for the presence of $S$. aureus. Moreover, E. aerogenes was isolated from CVC and urinary catheter cultures. Treatment with ciprofloxacin was started. The subsequent 10 days, the patient had progressive multisystem organ failure complicated by acute neutropenia, since microbiological cultures were negative. Despite antibiotic therapy, the patient died two months after surgery.

\section{Discussion}

Infections due to MDR Gram-negative rods are an emerging risk factor for increased mortality, and associated with high morbidity $(3,8,9,10,12)$. A baumannii has emerged as a potent nosocomial pathogen, provided with intrinsic resistance to the aminopenicillins and cephalosporins, and that over time acquired resistance to several antimicrobial agents, such as broad-spectrum -lactams, aminoglycosides, fluoroquinolones, tetracyclins, and, more recently, carbapenems $(3,8,9,10,12)$. Extensively drug-resistant (XDR) A. baumannii is a major threat in several critical care settings, associated with urinary tract infection, catheter-associated bloodstream infection, and ventilator-associated pneumonia $(3,8,9,10,12)$. The present clinical case had several clues for discussion. First, it showed that hospitalisation time as short as two days was shown to be enough to acquire an XDR nosocomial pathogen, as reported in other studies (3). The sources of $A$. baumannii infection were probably different and related to environmental contamination, mechanical ventilation, patient lifting equipment, door handles, mops, and keyboards, as also previously described in other clinical cases $(3,8,9,10,12)$. Second, after 3 days, A. baumannii strain was isolated in other anatomical sites, such as peritoneal liquid and wound due to surgery, indicating the presence of this pathogen in ICU, as well as in medical and surgical wards $(3,8,9,10,12)$. In fact, it is known that $A$. baumannii has the ability to survive and persist in the environment, in which, the production of biofilm on various implants, plays a key role to the survival, and a handicap for the treatment $(3,8,9,10,12)$. Third, this case report documents that $A$. baumannii strain acquired very rapidly the colistin-resistance, indicating rapidity of adaptation to antimicrobial agents and to the antibiotic therapies performed. Colistin have been used in recent years and remains to be the most active alternative to treat the VAP caused by XDR A. baumannii infections, although colistin-resistant $A$. baumannii strains have emerged, though still sporadic, in patients treated with this antimicrobial agent in monotherapy $(1,4)$. Different studies support the role of combination therapy of colistin with other antimicrobials in cases of multidrug-resistant (MDR) and XDR A. baumannii $(1,4,12)$. In the present clinical case, colistin was used in combination with vancomycin and imipenem. Therapeutic results were satisfactory in terms of clinical improvement, but this treatment was not sufficient to eradicate $A$. baumannii, which, rather, changed its antibiotic profile becom- 
ing all resistant, demonstrating a selection in vivo as Rolain and colleagues have previously described (11). Surprisingly, when antibiotic treatment was stopped, $A$. baumannii isolates changed to colistin phenotype. We can speculate (despite a study limitation due to the lack of a typing method to ascertain the clonality of the A. baumannii strains) that in vivo a transient resistance occurred, most probably due to the antibiotic pressure. Little information is known about the mechanisms that confer colistin resistance in A. baumannii; nevertheless, it appears to be multifactorial, chromosomally mediated, leading to modifications in bacterial lipopolysaccharide, capsular exopolysaccharide, and gene/protein expression profile $(2,6)$. More recently, it has been reported the emergence of the first plasmid-mediated polymyxin resistance mechanism, reporting a critical situation that requires an urgent global coordination against PDR Gram-negative bacteria (5).

Literature data regarding MDR/XDR A. baumannii involved in nosocomial infections are widely known $(3,8,9,10,12)$. The occurrence of MDR A. baumannii was related to the long-term use of wide spectrum antibiotics, putting patients under considerable risk of resistant infections $(3,8,9,10,12)$. Moreover, the decision to use invasive procedures and equipment must be decided on the basis of clinical situation of patient so that only necessary practices would be performed $(3,8,9,10,12)$. In addition, antimicrobial agents must be used for an optimal period at full dosage, preventing unnecessary and sub-optimal antibiotic use. It is important to treat infection caused by MDR/XDR microorganism as soon as possible with an appropriate antibiotic scheme, given that treatment options are limited. A compliance with the rules of hygiene, patient isolation, identification of germ sources, implementation of treatment strategies, and careful monitoring of antibiotic resistance evolution is also recommended.

\section{Conclusions}

Colistin is the last resort for treatment of MDR A. baumannii. Unfortunately, resistance to colistin has been reported all over the world. Colistin monotherapy is unable to prevent resistance, and combination therapy seems to be the best antimicrobial strategy against this mutable pathogen. Clinical observations like ours should assist to highlight the importance of prudent use of these antimicrobial agents and, therefore, well-designed clinical and pharmacological studies are necessary to prevent a widespread use of colistin not supported by careful judgments.

\section{References}

1. Cai Y, Chai D, Wang R, et al. Colistin resistance of Acinetobacter baumannii: clinical reports, mechanisms and antimicrobial strategies. J Antimicrob Chemother 2012;67:1607-15.

2. Geisinger E, Isberg RR. Antibiotic modulation of capsular exopolysaccharide and virulence in Acinetobacter baumanni. PLoS Pathog 2015;11:e1004691.

3. Gulen TA, Guner R, Celikbilek N, et al. Clinical importance and cost of bacteremia caused by nosocomial multi drug resistant Acinetobacter baumannii. Int J Infect Dis 2015;38:32-5.

4. Lesho E, Yoon EJ, McGann P, et al. Emergence of colistin-resistance in extremely drug-resistant Acinetobacter baumannii containing a novel pmrCAB operon during colistin therapy of wound infections. J Infect Dis 2013;208:1142-51.

5. Liu YY, Wang Y, Walsh TR, et al. Emergence of plasmid-mediated colistin resistance mechanism MCR-1 in animals and human beings in China: a microbiological and molecular biological study. Lancet Infect Dis 2016;16:161-8.

6. Lopez-Rojas R, McConnell MJ, Jimenez-Mejias ME, et al. Colistin resistance in a clinical Acinetobacter baumanni strain appearing after colistin treatment: effect on virulence and bacterial fitness. Antimicrob Agents Chemother 2013;57:4587-9.

7. Magiorakos AP, Srinivasan A, Carey RB, et al. Multidrug-resistant, extensively drug-resistant and pandrug-resistant bacteria: an international expert proposal for interim standard definitions for acquired resistance. Clin Microbiol Infect 2012;18:268-81.

8. Manchanda V, Sanchaita S, Singh NP. Multidrug resistant Acinetobacter. J Glob Infect Dis 2010;2:291-304.

9. Maragakis LL, Perl TM. Acinetobacter baumannii: epidemiology, antimicrobial resistance, and treatment options. Clin Infect Dis 2008;46:1254-63.

10. Michalopoulos A, Falagas ME. Treatment of Acinetobacter infections. Expert Opin Pharmacother 2010;11:779-88.

11. Rolain JM, Roch A, Castainer M, et al. Acinetobacter baumanni resistant to colistin with impaired virulence: a case report from France. J Infect Dis 2011;204:1146-7.

12. Vila J, Pachon J. Therapeutic options for Acinetobacter baumanni infections: an update. Expert Opin Pharmacother 2012;13: 2319-36. 